\title{
Quiste hidatídico hepático manejo laparoscópico: Reporte de caso
}

\author{
Robert William Campos-Guzmán¹, Oscar Altamirano-Narrea ${ }^{1}$ Julio Romero-Palomino²
}

\section{RESUMEN}

Se reporta el caso de un paciente de sexo masculino de 32 años de edad, con antecedente de cirugía torácica por Quiste Hidatídico Pulmonar a los 9 años de edad; que ingresa al Servicio de Cirugía General y Digestiva del Hospital II Lima Norte Callao Luis Negreiros Vega, con tiempo de enfermedad de 1 año aproximadamente, refiriendo dolor abdominal tipo opresivo de leve a moderada intensidad a predominio de hemiabdomen superior, principalmente hipocondrio derecho y sensación de masa en dicha zona. Concomitantemente refiere el antecedente de cirugía previa en región torácica y con antecedente epidemiológico positivo. Luego de la evaluación clínica por el equipo de cirugía de consultorio externo y de la observación e informe de la Tomografía Espiral Multicorte en la que se observa lesión bien definida de aspecto multiquístico en segmento IV y $\mathrm{V}$, que muestra realce periférico luego de administración de contraste; asociado a Western Blot positivo, se decide programación quirúrgica. Se realizó cirugía laparoscópica que consistió en destechamiento del quiste, aspiración y lavado del interior con hipersodio (CINa 20\%) y colocación de drenaje mixto. El paciente presento en el postoperatorio fistula biliar que se resolvió en 5 semanas; con inicio de tolerancia oral el primer día postoperatorio y controles de perfil hepático dentro de rangos normales al alta de cirugía que se realizó al 4 día post operatorio.

(Horiz Med 2015; 15(4): 74-77)

Palabras clave: Quiste hidatídico hepático; cirugía laparoscópica.(Fuente: DeCS BIREME).

Hepatic hydatid cyst, laparoscopic management. Case report

\section{ABSTRACT}

We report a case of male patient of 32 years old; with a history of thoracic surgery for hydatid cyst at 9 years of age; admission was at the general surgery Service of the Hospital II Lima Norte Callao Luis Negreiros Vega, with a history of illness of 1 year, referring abdominal pain oppressive predominance of the upper abdomen, especially on right upper quadrant. Refers concomitantly history of previous surgery in thoracic region and positive epidemiological history. After clinical evaluation by the staff of surgery, outpatient clinic and the observation and reporting of CT in which well-defined lesions in segment IV and V multicystic appearance and lesser sac showing peripheral enhancement it is observed after administration contrast; associated with positive Western Blot, surgical treatment is decided. Laparoscopic Surgery was performed consisting of cyst aspiration drainage and washing the interior with hipersodio ( $20 \% \mathrm{ClNa}$ ) and placement of laminar drain was done. Presented a postoperative biliary fistula that was resolved in five weeks, beginning of oral tolerance on the first postoperative day and liver function controls within normal ranges discharge was performed four days after surgery. (Horiz Med 2015; 15(4): 74-77)

Key words: Hepatic hydatid cyst; Laparoscopic surgery. (Source: MeSH NLM).

Cirujano Asistente del Servicio de Cirugía General del Hospital II Lima Norte Callao Luis Negreiros Vega

Médico Residente Cirugía General, Hospital II Lima Norte Callao Luis Negreiros Vega 


\section{INTRODUCCIÓN}

La Hidatidosis es una zoonosis causada por el estadio larvario del Céstode equinococcus granulosus. Es una enfermedad endémica de los países del Medio Oriente, los países mediterráneos, y de Latinoamérica.

El turismo hace que ahora su patrón de distribución sea más amplio, incluso en países desarrollados (1).

El parásito requiere de diferentes hospederos para completar el ciclo de vida: los hospederos definitivos son el perro doméstico y otros cánidos silvestres como lobos o chacales, donde desarrolla la forma adulta o estrobilar; y los hospederos intermediarios, son principalmente ovinos pero también, caprinos, cerdos, bovinos, guanacos, etc. en los cuales desarrolla la forma o fase larvaria o metacestode (2).

El hombre adquiere la infección a través de la ingestión de agua o de alimentos contaminados con los huevos del parásito, o por el contacto estrecho y descuidado con perros parasitados (dejarse lamer, darle besos) $(2,3)$.

En este sentido, la niñez es la etapa de la vida donde generalmente se produce la infección. Al igual que en el ganado, en el intestino delgado se produce la disolución de la cubierta de los huevos del parásito, y se liberan embriones que atraviesan la mucosa intestinal y pasan a la circulación portal para llegar a los diferentes órganos, principalmente el hígado, aunque pueden llegar al pulmón y menos frecuentemente a otros órganos como cerebro, riñones, bazo, hueso, etc (3).

Los Quiste Hidatídicos son a menudo de localización hepática (50-80\%) y pulmonar (5-30\%), pero también se pueden encontrar en otras localizaciones $(1,4)$.

El tratamiento depende de las características de la lesión quística, el paciente y si existen contraindicaciones para la cirugía, que sigue siendo el Gold estándar terapéutico.

El tratamiento médico se caracteriza por el uso de benzimidazoles, preoperatoriamente con en el postoperatorio (5).

La punción, aspiración, inyección y reaspiración (PAR), han demostrado ser eficaz en el tratamiento de los quistes hidatídicos no complicados (6).

La CPRE ha sido reconocida como la herramienta que brinda el mayor enfoque en la Hidatidosis hepática complicada, siendo a la vez diagnóstica y terapéutica (7).

\section{CASO CLINICO}

Paciente de sexo masculino de 32 años de edad, con antecedente epidemiológico de zona endémica, sin otros antecedentes médicos y con cirugía previa de cirugía torácica por quiste hidatídico pulmonar a los 9 años de edad.

Ingresa al Servicio de Cirugía del Hospital II Lima Norte Callao Luis Negreiros Vega, con tiempo de enfermedad de 1 año aproximadamente, refiriendo dolor abdominal tipo opresivo de leve a moderada intensidad a predominio de hemiabdomen superior, principalmente en hipocondrio derecho; asociado a sensación de masa en dicha zona, niega síntomas asociados, como hiporexia, sensación nauseosa, vómitos, o cambios en el hábito defecatorio.

En el examen clínico al momento de la evaluación se encontraba en buen estado general, y adecuado estado de hidratación, entre los hallazgos significativos en el abdomen este se encontraba no distendido y se palpaba masa a nivel de hipocondrio derecho de bordes regulares, de aproximadamente $5 \times 5 \mathrm{~cm}$, no elevada, no móvil, no dolorosa a la palpación, no signos peritoneales al momento del examen.

Con estos hallazgos la Tomografía Espiral Multicortes (Figuras 1 y 2) mostraba: en porción derecha del domo hepático, calcificación $(30 \times 25 \mathrm{~mm})$, así como lesión focal bien definida de aspecto multiquístico en segmentos IV $Y$ V $(87 \times 70 \mathrm{~mm})$ y transcavidad de los epiplones $(70 \times 45 \mathrm{~mm})$, que muestran discreto realce periférico luego de la administración del contraste. 


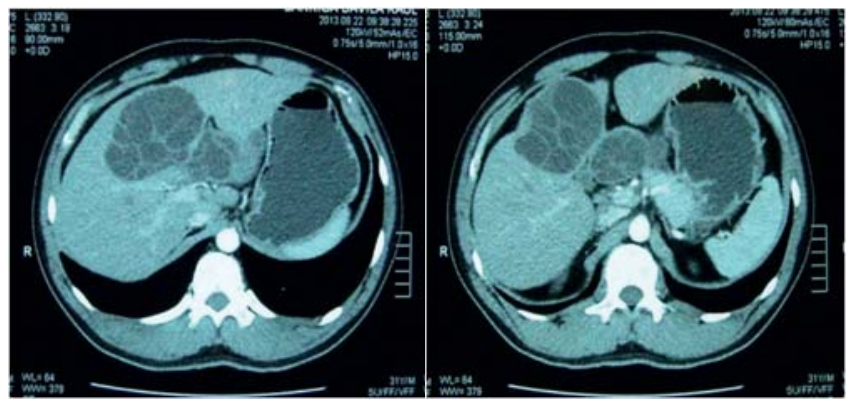

Figura 1. Lesión focal Figura 2. Lesión focal bien definida de aspecto bien definida de aspecto multiquístico en segmento IV. transcavidad de los epiplones que muestran realce periférico.

Asociado a una prueba de Western Blot positivo para Hidatidosis, se decide realizar cirugía laparoscópica programada, previo tratamiento de un mes con albendazol a $10 \mathrm{mg} / \mathrm{Kg} /$ día. Se evidencia en el intraoperatorio adherencias laxas y firmes de epiplón a vesícula biliar y estructura quística de $20 \times 15 \mathrm{~cm}$. de diámetro, que comprende los segmentos IVA, IVB y V, de paredes gruesas, conteniendo liquido turbio y biliar, además de vesículas hijas múltiples siendo la mayor de $6 \mathrm{~cm}$. aproximadamente; dentro de la cavidad del mismo, se evidencia comunicación con otro quiste más pequeño de $8 \times 6 \mathrm{~cm}$., dependiente aparentemente de segmento I, que contiene abundantes vesículas, las cuales son removidas y absorbidas en su totalidad (Figura 3 y 4 ).

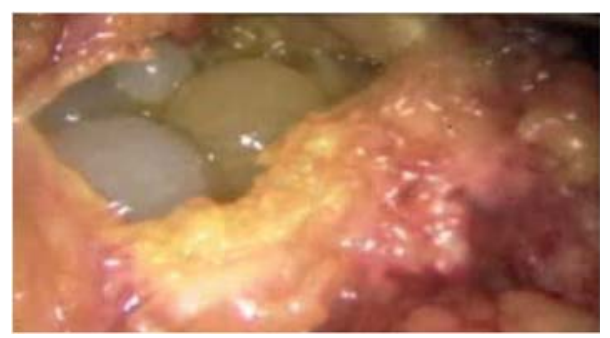

Figura 3. Apertura de pared quística y visualización de vesículas hijas.

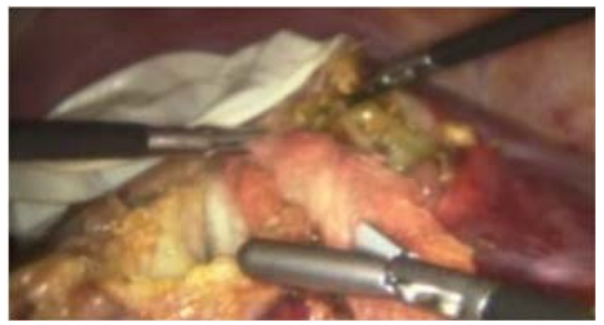

Figura 4. Legrado con las gasas embebidas con hipersodio en toda la pared quística, luego introducidas dentro de la bolsa colectora.

Se realizó liberación de adherencias, colocación de gasas embebidas con hipersodio, aspiración de contenido de quiste, instilación de solución hipertónica a cavidad de quiste, se deja solución por 5 minutos, y posteriormente se procedió a destechamiento de quiste, verificación de hallazgos, aspiración de contenido con remoción completa de vesículas, luego se realizó raspado con gasa con hipersodio en lecho quístico y finalmente colocación de drenes (laminar y tubular), en espacio de Morrison y en lecho de cavidad quística destechada, y colocación de epiplón mayor en dicha cavidad.

Paciente cursa en el postoperatorio inmediato con evolución favorable de los parámetros clínicos, afebril, con secreción sero-biliosa a través de drenaje mixto. Inicia tolerancia a las 24 horas de su postoperatorio y es dado de alta al 4 día, con gasto bajo por drenaje mixto de iguales características que su estancia hospitalaria. En consultorio externo se diagnosticó fistula biliar establecida, cuyo seguimiento se llevó a cabo hasta la resolución del mismo en 5 semanas, desde el postoperatorio, los controles ecográficos y séricos fueron normales (Figura 5 y 6).

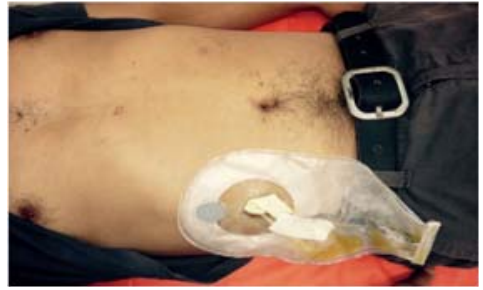

Figura 5. Paciente durante el seguimiento en consultorio externo de la cirugía y fistula biliar instaurada.

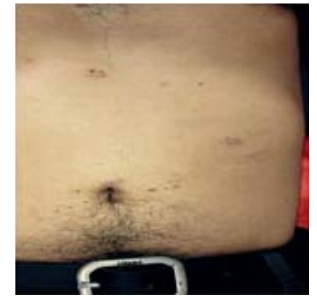

Figura 6. Paciente al alta de consultorio externo de cirugía.

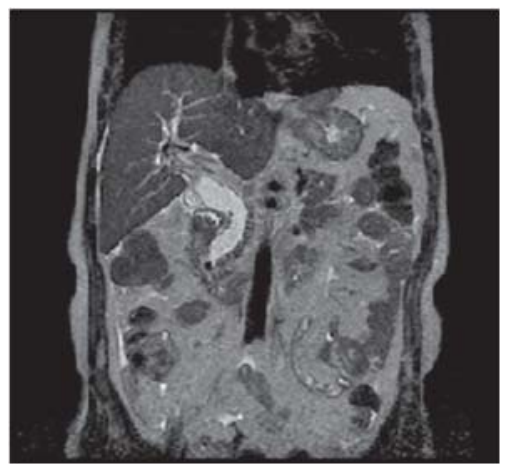

Figura 7. RMN de hígado y vías biliares, no se evidencia recidiva de enfermedad a los 6 meses de control post operatorio. 


\section{DISCUSIÓN}

El tratamiento de los quistes hidatídicos del hígado siempre ha sido un reto para el cirujano, debido a las potenciales complicaciones como el choque anafiláctico o la diseminación intrabdominal. Sin embargo, grupos de trabaj o en zonas endémicas del país como en ciudades de la sierra central han adquirido enorme experiencia en estos casos, con resultados óptimos. El uso de técnicas laparoscópicas, nos permite ingresar progresivamente a nuevas patologías para su resolución por invasión mínima, tal es el caso de las hernioplastías, tratamiento del reflujo gastroesofágico, esplenectomía, cirugía de la obesidad mórbida y ahora el tratamiento laparoscópico de los quistes hidatídicos hepáticos.

Tomando en cuenta las consideraciones antes mencionadas, hemosusado la técnica laparoscópica, aplicando los principios de la cirugía hidatídica hepática tradicional, que incluye la inactivación de los escólices, prevención de la filtración, eliminación de la membrana germinativa; acompañado de un adecuado manejo de la cavidad residual.

La mayor desventaja de la técnica laparoscópica es la falta de adecuadas medidas preventivas para evitar la filtración del contenido hidatídico, especialmente bajo la alta presión intra-abdominal inducida por el neumoperitoneo. El paso más peligroso es la punción inicial y la aspiración del contenido quístico y es por tal razón que se utiliza en el pre y post-operatorio Albendazol $(10 \mathrm{mg} / \mathrm{kg} /$ día), 30 días en el preoperatorio y dos meses en el postoperatorio, esquema de tratamiento utilizado en el presente caso. Para prevenir una eventual filtración se rodea el sitio a punzar con gasas impregnadas con hipersodio (CINa 20\%) y se utiliza un doble mecanismo de aspiración.

Las técnicas laparoscópicas reportadas en el manejo del quiste hidatídico hepático, se pueden dividir en dos grandes grupos, aquellas que realizan extirpación parcial o total de la envoltura periquística y las que efectúan solamente evacuación de la membrana germinativa. La mortalidad relacionada a esta patología, está asociada principalmente a la anafilaxia, y se encuentra favorecida por la no administración preoperatoria de benzimidazoles, y a la no prevención de la filtración.

\section{REFERENCIAS BIBLIOGRÁFICAS}

1. Mohammad Reza Motie, Mohammad Ghaemi, Mohsen Aliakbarian; Elena Saremi; "Study of the Radical vs. Conservative Surgical Treatment of the Hepatic Hydatid Cyst: A 10-Year Experience"; Indian J Surg (November-December 2010) 72(6):448-452.

2. Sociedad Argentina de Infectología, Sociedad Argentina de Pediatría Sociedad Argentina de Medicina Filial Argentina de la Asociación Internacional de Hidatidología; "Enfermedades Infecciosas/ Hidatidosis, "Guía para el equipo de salud"; Argentina; Guía para el equipo de salud Nro 11; Marzo/2012.

3. Pedro Silva Vaz, Eduardo Pereira, Sergiu Usurelu, Ana Monteiro, Ana Caldeira, Gina Melo, Rui Sousa, António Gouveia and Arnandina Loureiro; "Hepatic hydatid cyst: a non-surgical approach"; Brazil; Revista da Sociedade Brasileira de Medicina Tropical 45(6):774776, Nov-Dec, 2012.

4. Dr. Gustavo Salinas Sedó, Dr. Carlos Velásquez Hawkins, Dra. Lil Saavedra Tafur; Tratamiento laparoscópico de los Quistes hidatídicos hepáticos; Perú, 306 Revista Gastroenterología Peruana 21: 306 - 311, 2001.

5. Eduardo Miranda1, Elizabeth Sánchez1, César Náquira1,2, José Somocursio3, Eduardo Ayala1, Gilberto Miranda4; Evaluación de una prueba de aglutinación de látex para el diagnóstico serológico de la Equinococosis quística; Rev Perú Med Exp Salud Publica. 2009; 26(2): 198-202.

6. Pedro Pinto, Claudio Vallejos, Eduardo Cruces, José Lobos, Francisco Hernández, Marcelo Ríos, Sr José Gálvez; Tratamiento Laparoscópico del Quiste Hidatídico Hepático; Revista chilena de Cirugía Vol. 63, n 4, Santiago agosto 2011.

7. Dr. Pedro Pinto G.; Actualización en el diagnóstico y tratamiento de la hidatidosis hepática; Revista chilena de Cirugía Vol. 60, n6, Santiago diciembre 2008, pág. 561-566.

\section{Fuentes de financiamiento}

Este artículo ha sido financiado por los autores.

\section{Conflictos de interés}

Los autores declaran no tener conflicto de interés en la presentación de este artículo.

\section{Correspondencia:}

Robert William Campos-Guzmán

Teléfono: Celular: 959-691-200 rpm \# 065090

Dirección: Calle Petronila Álvarez 107 San Miguel.

Correo electrónico: drwilliamcamposguzman@ gmail.com

Recibido: 22 de Julio de 2015

Aprobado: 08 de Agosto de 2015 